\title{
Phenotypic Variations in Camptotheca Decaisne
}

\author{
Shiyou Li*
}

National Center for Pharmaceutical Crops, Arthur Temple College of Forestry and Agriculture, Stephen F. Austin State University, Nacogdoches, TX 75962, USA

\begin{abstract}
Variations in shape, size, color, and texture of fruits, shape and size of cotyledons, shape, venation pattern, surface and margin characters of mature leaves of 40 populations (either living plants or herbarium specimens) representing all known taxa of Camptotheca have been analyzed within and between populations. A majority of the observed phenotypic variations for most morphometric measures was among populations. This is probably due largely to selfing and related matings common to both natural and cultivated populations, resulting in the current population structure that now displays a highly fragmented distribution pattern. Trees of different generations from the same seed source but growing in different locations demonstrated minimal variations in both means and frequency distributions of leaf vein number, fruit length, fruit colors, and cotyledon vein number. The trees of different taxa even growing in the same location have significant difference in main characters particularly fruit color and texture. The key diagnostic characters for the identification of taxa of Camptotheca are leaf shape, venation type, vein number, stoma size and frequency, outer stomatal rim, subsidiary cell number, and gland size; cotyledon shape, venation type, and vein number; and the fruit surface texture, disc, length, and color.
\end{abstract}

Keywords: Camptotheca, cotyledon shape, fruit color, fruit length, fruit surface texture, gland length, leaf surface, leaf vein number, leaf venation type, phenotypic variations,

\section{INTRODUCTION}

Evolutionary ecologists have stressed the importance of understanding the expression and the components of phenotypic variation among and within populations [1-6] and have proposed that a hierarchical perspective may provide insights into the evolutionary history of a species [3]. Phenotypic variations of the Camptotheca or any species of the genus have never been analyzed. The primary objectives of this study are: (1) to investigate the pattern of phenotypic variation within and among populations; (2) to determine whether these traits examined are taxon specific and can be used as diagnostic key characters in the genus; and (3) to clarify taxonomic and systematic relationships.

\section{MATERIALS AND METHODS}

\section{Plant Materials}

General information on the plant materials collected from 1992 to 1995 for this study is presented in (Table 1). The letters $\mathrm{A}, \mathrm{B}, \mathrm{L}$, and $\mathrm{Y}$ followed by numbers designate populations. The letter A refers to the plant materials of C. acuminata var. acuminata collected directly from China: A1-9 represents the dominant seed sources in current plantations in southern China. A10-16 refers to herbarium specimens. The letter B refers to the materials of C. acuminata var. acuminata collected from the United States: B1-6 represents mature trees in the country (in 1995). B3a and B3b were collected from the same tree San Antonio, Texas in 1993 and

*Address correspondence to this author at the National Center for Pharmaceutical Crops, Arthur Temple College of Forestry and Agriculture, Stephen F. Austin State University, Nacogdoches, TX 75962, USA; Tel: 936-4682071; Fax: 936-468-7058; E-mail: lis@sfasu.edu
1995, respectively, and B6a and B6b were collected from the same tree in Huntington, California in 1992 and 1995, respectively. Three of these samples represent first generation (B2) and the third generation (B3 and B4) from the same ancestor see Fig. (1). The letter $\mathrm{L}$ refers to populations of $C$. lowreyana: L1, L4, L5, and L6 are herbarium specimens, while L2 and L3 were collected from China. The letter Y refers to population of C. yunnanensis: $\mathrm{Y} 1$ was collected in China, and Y2 is the herbarium specimen. In addition, the only sample of $C$. acuminata var. tenuifolia is the type specimen. The data on C. acuminata var. rotundifolia are drawn from the original description.

Climatic data including annual precipitation and mean January temperatures were determined for each collection site from the local records (Table 2).

\section{Fruit and Cotyledon Shapes and Sizes}

All fruit from herbarium specimens were measured. For our collections, fruits from six individuals were measured, germinated, and grown in the same environments for fruit and seedling phenotypic analyses. For morphometric analysis, length and maximum width of 64 randomly selected mature dry fruit from six trees were measured. For seedling analysis, sample size was dependent on the seedlings available. Cotyledon venation type, lateral vein number, blade length, blade width at widest point, and widths at $1 / 5$ distance from the apex and the base were measured at the age of three months.

\section{Fruit Color and Texture}

Color is usually diagnostic in plant taxonomy. Careful color evaluation is desirable for botanical descriptions. A 
Table 1. Description of the populations of Camptotheca surveyed $(*$ Tree age $=$ parent tree age when seeds were collected; $* *$ Seedling age = age in 1997; ---- no data available/unapplicable; $A=$ collections of $C$. acuminata from $C$ hina; $B=$ collections of C. acuminata from the United States; $\mathrm{L}=$ C. lowreyana; $\mathrm{Y}=C$. yunnanensis; A1-9, B1-6, L1-3, and Y1 = living collections; rest $=$ herbarium specimens).

\begin{tabular}{|c|c|c|c|c|c|c|}
\hline Seed Source & Origin & $\begin{array}{c}\text { Mature Tree } \\
\text { Population } \\
\text { Size }\end{array}$ & $\begin{array}{c}\text { Seed } \\
\text { Collection } \\
\text { Year }\end{array}$ & $\begin{array}{l}\text { Tree } \\
\text { Age * } \\
\text { (year) }\end{array}$ & $\begin{array}{l}\text { Germinated } \\
\text { Seedling } \\
\text { Number }\end{array}$ & $\begin{array}{l}\text { Seedling } \\
\text { Age ** } \\
\text { (year) }\end{array}$ \\
\hline $\begin{array}{l}\text { C. acuminata var. acuminata } \\
\text { A1 a. Dakengshan, Huaiji, Guangdong, China } \\
\text { A1b. Dakengshan, Huaiji, Guangdong, China } \\
\text { A2. Gushui, Guangning, Guangdong, China } \\
\text { A3. Pengzhou, Sichuan, China } \\
\text { A4. Jintang, Sichuan, China } \\
\text { A5. China (F.W. Schumacher Co, MA) } \\
\text { A6. Nanjing, Jiangsu, China } \\
\text { A7. China (Lawyer Nursey, MT) } \\
\text { A8. Xuancheng, Anhui } \\
\text { A9. Zhejiang, China (Lawyer Nursey, MT) } \\
\text { A10. Emeishan, Sichuan, China (W. C. Cheng 2231) } \\
\text { A11. Nanjing, Jiangsu, China (K. S. Chow 80271) } \\
\text { A12. Hubei, China (A. Henry 7606) } \\
\text { A13. Guangzhou, Guangdong, China (H. G. Yip 431) } \\
\text { A14. Yunghsien, Guangxi, China (Steward \& Cheo 1180) } \\
\text { A15. Guilin, Guangxi, China (Wan \& Chow 79088) } \\
\text { A16. Lushan, Jiangxi, China (David 866, isotype) } \\
\text { A17. Botanical Garden, Guangzhou, China } \\
\text { A18. Botanical Garden, Xian, Shaanxi, China } \\
\text { A19. Changan, Shaanxi, China } \\
\text { A20. Yangling, Shaanxi, China } \\
\text { A21. China (unknown) }\end{array}$ & \begin{tabular}{l} 
Cultivated \\
Cultivated (same with A1a) \\
Cultivated (same with A1) \\
Cultivated \\
Cultivated \\
Cultivated \\
Cultivated \\
Cultivated \\
Cultivated \\
Cultivated \\
\hdashline--- \\
Cultivated \\
\hdashline--- \\
Cultivated \\
\hdashline--- \\
Cultivated \\
---- \\
Cultivated \\
Cultivated \\
Cultivated \\
Cultivated \\
Cultivated
\end{tabular} & \begin{tabular}{l}
24 \\
24 \\
18 \\
c. 300 \\
c. 500 \\
\hdashline--- \\
40 \\
\hdashline--- \\
---- \\
---- \\
---- \\
---- \\
---- \\
\hdashline--- \\
---- \\
--- \\
--- \\
3 \\
2 \\
20 \\
5 \\
-----
\end{tabular} & $\begin{array}{l}1994 \\
1996 \\
1994 \\
1994 \\
1994 \\
1994 \\
1994 \\
1994 \\
1995 \\
1991 \\
1930 \\
1980 \\
1885-88 \\
1981 \\
1933 \\
1979 \\
1868 \\
1996 \\
1996 \\
1996 \\
1996 \\
1996\end{array}$ & $\begin{array}{l}18 \\
20 \\
18 \\
>10 \\
>10 \\
>10 \\
25 \\
>10 \\
>10 \\
>10 \\
---- \\
---- \\
---- \\
---- \\
---- \\
---- \\
---- \\
18 \\
8 \\
25 \\
25 \\
-----\end{array}$ & \begin{tabular}{|l}
63 \\
33 \\
154 \\
0 \\
22 \\
0 \\
850 \\
220 \\
350 \\
26 \\
--- \\
---- \\
---- \\
---- \\
---- \\
---- \\
--- \\
0 \\
24 \\
52 \\
65 \\
----
\end{tabular} & $\begin{array}{l}3 \\
1 \\
3 \\
-3--- \\
3 \\
--- \\
3 \\
3 \\
3 \\
6 \\
---- \\
--- \\
---- \\
---- \\
---- \\
---- \\
---- \\
1 \\
1 \\
1 \\
1 \\
-----\end{array}$ \\
\hline $\begin{array}{l}\text { C. acuminata var. acuminata } \\
\text { B1. Chico, CA, USA (east tree) } \\
\text { B2. Chico, CA, USA (west tree) } \\
\text { B3a. San Antonio, TX, USA } \\
\text { B3b. San Antonio, TX, USA } \\
\text { B4. Kingwood, TX, USA } \\
\text { B5. Summerville, SC, USA } \\
\text { B6a. Huntington, San Marino, CA, USA } \\
\text { B6b. Huntington, San Marino, CA, USA } \\
\text { B7a. SFA Arboretum, Nacogdoches, TX, USA } \\
\text { B7b. SFA Arboretum, Nacogdoches, TX, USA } \\
\text { B8. SFA Exper. Forest, Nacogdoches, TX, USA } \\
\text { C. acuminata var. rotundifolia } \\
\text { C. acuminata var. tenuifolia } \\
\text { C. lowreyana } \\
\text { L1. Lianxian, Guangdong, China (Gao 50863, type) } \\
\text { L2. Shiying, Huaiji, Guangdong, China } \\
\text { L3. Dakengshan, Huaiji, Guangdong, China } \\
\text { L4. Tongzhong, Huaiji, Guangdong, China (Tsang 23130) } \\
\text { L5. Lechang, Guangdong, China (Jiang 1429) } \\
\text { L6. Yaoshan, Guangdong, China (Wang 40005) } \\
\text { C. yunnanensis } \\
\text { Y1. Mengyang, Xishuangbanna, Yunnan, China } \\
\text { Y2. Simao, Yunnan, China (Henry 13433) } \\
\text { Y3. Yangbi, Yunnan, China } \\
\text { Y4. Kunming, Yunnan, China }\end{array}$ & $\begin{array}{l}\text { Cultivated (from Jiangsu, China) } \\
\text { Cultivated (same with B1) } \\
\text { Cultivated (parent tree from B2) } \\
\text { The same tree with B3a } \\
\text { Cultivated (same with B3) } \\
\text { Cultivated (from Honolulu) } \\
\text { Cultivated (local source) } \\
\text { The same tree with B6a } \\
\text { Cultivated } \\
\text { Cultivated (same tree with B7a) } \\
\text { Cultivated } \\
\text { Natural (?) } \\
\text { Natural } \\
\text { Natural } \\
\text { Natural } \\
\text { Natural } \\
\text { Natural } \\
\text { Natural } \\
\text { Natural } \\
\text { Natural } \\
\text { Cultivated } \\
\text { Cultivated } \\
\text { Cultivated }\end{array}$ & $\begin{array}{l}1 \\
1 \\
1 \\
1 \\
1 \\
1 \\
1 \\
1 \\
1 \\
1 \\
1 \\
1 \\
---- \\
1 \\
--- \\
21 \\
28 \\
---- \\
---- \\
---- \\
\text { c. } 10 \\
---- \\
\text { c. } 250 \\
>100\end{array}$ & $\begin{array}{l}1992 \\
1992 \\
1993 \\
1995 \\
1994 \\
1994 \\
1992 \\
1995 \\
1996 \\
1997 \\
1996 \\
----- \\
1996 \\
1930 \\
1994 \\
1994 \\
1933 \\
1928 \\
1936 \\
1996 \\
---- \\
1994 \\
1996\end{array}$ & $\begin{array}{l}58 \\
58 \\
12 \\
14 \\
12 \\
24 \\
29 \\
32 \\
5 \\
6 \\
5 \\
----- \\
->10 \\
>50 \\
>100 \\
----- \\
--- \\
--- \\
>80 \\
---- \\
46 \\
>30\end{array}$ & \begin{tabular}{|l}
---- \\
---- \\
20 \\
125 \\
20 \\
50 \\
---- \\
134 \\
c. 600 \\
---- \\
0 \\
---- \\
12 \\
---- \\
22 \\
35 \\
---- \\
---- \\
--- \\
54 \\
---- \\
20 \\
11
\end{tabular} & \begin{tabular}{|l}
---- \\
4 \\
4 \\
3 \\
3 \\
--- \\
2 \\
1 \\
---- \\
--- \\
--- \\
2 \\
--- \\
3 \\
3 \\
---- \\
---- \\
1 \\
---- \\
3 \\
1
\end{tabular} \\
\hline
\end{tabular}

general description of color is inadequate today. To avoid human visual errors, remote sensing technology was applied in the fruit color analyses. Thirty dry fruit from each of 17 populations of Camptotheca were selected for chroma and surface texture analysis using remote sensing technology. The procedure involves the following steps: (1) optical digitization using a Sony DXC 327A video camera; (2) recording the digitized image using a "frame grabber" video board (video 7 band) installed in an HP 9000 Model 720 workstation; (3) image processing using ERDAS Imagine 8.1 (ERDAS Inc., 1990) software normalized for contrast and brightness by setting the Histogram Contrast Adjustment to a linear curve indexed from 0 to 255 for all three channels (red, green, and blue) Fig. (1); (4) definition of the AOI (area of interest) of each fruit manually; (5) adding AOIs to each seed signature and then merging signatures through a classification protocol; and (6) displaying statistics (means and standard deviations) for all three (red, green, and blue) color channels for all seed signatures. Diagnostic differences in seed chroma were recorded indicating that multi-spectral analysis may be a productive area for further research in classifying visual indicators in plant taxonomy. 


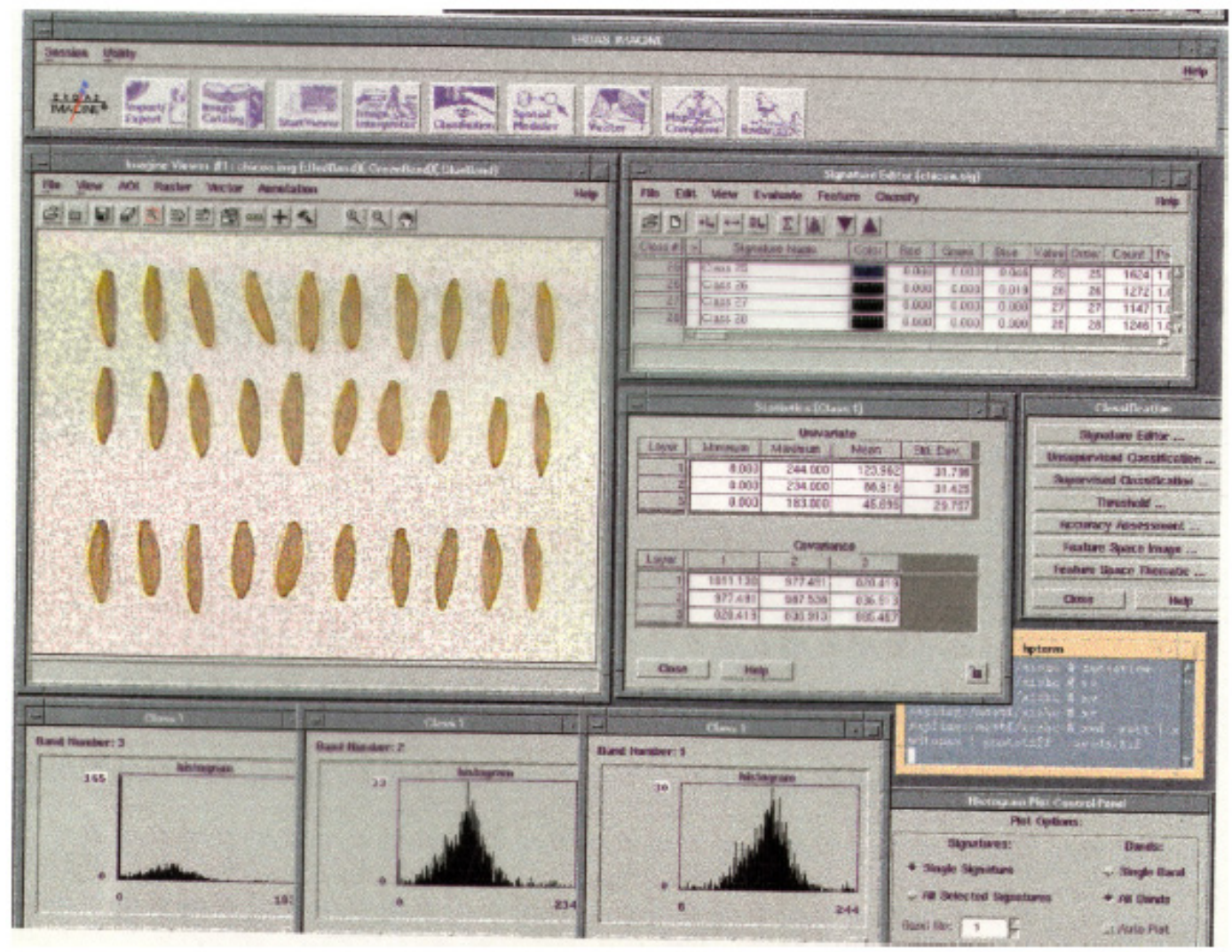

Fig. (1). Analysis of fruit color and size of Camptotheca using ERDAS Imagine 8.1 (ERDAS Inc., 1990).

\section{Leaf Shapes, Venation Patterns, and Margin Characters}

Each sample (64 mature leaves when possible) was selected from three trees for each of 17 populations/specimens of C. acuminata var. acuminata, six populations of $C$. lowreyana, and two of C. yunnanensis. Leaf shape, vein number, blade length, blade width at widest point, and blade widths at $1 / 5$ the distance from the apex and from the base were recorded for each leaf. Leaf margin characters include three categories: serrate, slightly serrate, and entire was measured for all samples. Both parent trees and seedlings were measured when possible.

\section{Statistical Analysis}

Phenotypic variations of populations were compared using ANOVA and Tukey's Studentized Range (HSD) Test for each of the quantitative measures. Means, standard deviations, and variation ranges of all characters were calculated for all samples. Statistical significance levels employed are $0.05,0.01$, and 0.001 . Frequency distributions of all characters in each sample are also presented because means-based methods tend to obscure overlap. Pearson correlation coefficients were calculated for major measures. Statistical analyses employed SAS version 6.03 (SAS Institute 1990) at SFASU.

\section{RESULTS}

\section{Fruits (Seeds)}

All observed samples of C. acuminata (var. acuminata, var. rotundifolia, and var. tenuifolia) have a rugose surface and thick disc when dry. Fruit of C. lowreyana and C. yunnanensis have a smooth surface and thin disc relative to $C$. acuminata (Table 3). C. lowreyana, with a mean fruit length of $29.02 \mathrm{~mm}$, similar to C. acuminata var. tenuifolia (mean length: $29.80 \mathrm{~mm}$ ) has larger fruit than either $C$. acuminata var. acuminata (22.54 mm) (Fig. 2) or C. yunnanensis (22.96 $\mathrm{mm}$ ). A frequency distribution of fruit length shows the same relationships Fig. (3). The ratio of fruit length by width also displayed significant differences among species: C. lowreyana, C. acuminata var. acuminata, and C. yunnanensis have mean ratios of 5.08, 4.18, and 3.61, respectively. In fact, ratio differences are largely contributed by the length differences among species.

A quantitative description of fruit color provides an important and objective measure of phenotypic variation and is diagnostic in taxon identification of Camptotheca. C. yunnanensis displays significant differences in red, green, and blue chroma from $C$. acuminata and $C$. lowreyana both in terms of means and distribution frequencies for the number of fruit measured (Table 4), Fig. (4).

\section{Cotyledons}

Venation type is constant within species, but different between C. lowreyana (pinninerved) and C. acuminata var. acuminata or C. yunnanensis (pinnipalmate). Vein number of the cotyledon is also an important character in distinguishing C. lowreyana (mean vein number: 6.91 ) from both $C$. acuminata var. acuminata (3.48) and C. yunnanensis (3.21) (Table 5); Fig. (5). Vein number is also independent of cotyledon size, the latter often varying with environment. 
Table 2. Site descriptions of the populations of Camptotheca surveyed (---- no data available).

\begin{tabular}{|c|c|c|c|c|c|c|}
\hline Population/Location & Latitude & Longitude & $\begin{array}{c}\text { Altitude } \\
\text { (m) }\end{array}$ & $\begin{array}{c}\text { Lowest } \\
\text { Temperature } \\
\left({ }^{\circ} \mathrm{C}\right)\end{array}$ & $\begin{array}{c}\text { Mean January } \\
\text { Temperature } \\
\left({ }^{\circ} \mathrm{C}\right)\end{array}$ & $\begin{array}{c}\text { Annual } \\
\text { Precipitation } \\
\text { (mm) }\end{array}$ \\
\hline $\begin{array}{l}\text { C. acuminata var. acuminata } \\
\text { B1. Chico, CA, USA (east tree) } \\
\text { B2. Chico, CA, USA (west tree) } \\
\text { B3a, b. San Antonio, TX, USA } \\
\text { B4. Kingwood, TX, USA } \\
\text { B5. Summerville, SC, USA } \\
\text { B6a, b. Huntington, San Marino, CA, USA } \\
\text { B7a, b. SFA Arboretum, Nacogdoches, TX, USA } \\
\text { B8. SFA Exper. Forest, Nacogdoches, TX, USA } \\
\text { C. acuminata var. rotundifolia } \\
\text { C. acuminata var. tenuifolia } \\
\text { C. lowreyana } \\
\text { L1. Lianxian, Guangdong, China } \\
\text { L2. Shiying, Huaiji, Guangdong, China } \\
\text { L3. Dakengshan, Huaiji, Guangdong, China } \\
\text { L4. Tongzhong, Huaiji, Guangdong, China } \\
\text { L5. Lechang, Guangdong, China } \\
\text { L6. Yaoshan, Guangdong, China } \\
\text { C. yunnanensis } \\
\text { Y1. Mengyang, Xishuangbanna, Yunnan, China } \\
\text { Y2. Simao, Yunnan, China } \\
\text { Y3. Yangbi, Yunnan, China } \\
\text { Y4. Kunming, Yunnan, China }\end{array}$ & $\begin{array}{l}39^{\circ} 42^{\prime} \mathrm{N} \\
39^{\circ} 42^{\prime} \mathrm{N} \\
29^{\circ} 25^{\prime} \mathrm{N} \\
29^{\circ} 55^{\prime} \mathrm{N} \\
33^{\circ} 02^{\prime} \mathrm{N} \\
34^{\circ} 07^{\prime} \mathrm{N} \\
31^{\circ} 38^{\prime} \mathrm{N} \\
? \\
24^{\circ} 30^{\prime} \mathrm{N} \\
24^{\circ} 47^{\prime} \mathrm{N} \\
24^{\circ} 28^{\prime} \mathrm{N} \\
23^{\circ} 50^{\prime} \mathrm{N} \\
24^{\circ} 05^{\prime} \mathrm{N} \\
25^{\circ} 12^{\prime} \mathrm{N} \\
24^{\circ} 30^{\prime} \mathrm{N} \\
22^{\circ} 10^{\prime} \mathrm{N} \\
23^{\circ} 00^{\prime} \mathrm{N} \\
25^{\circ} 45^{\prime} \mathrm{N}\end{array}$ & $\begin{array}{l}121^{\circ} 47^{\prime} \mathrm{W} \\
121^{\circ} 47^{\prime} \mathrm{W} \\
98^{\circ} 30^{\prime} \mathrm{W} \\
94^{\circ} 50^{\prime} \mathrm{W} \\
80^{\circ} 12^{\prime} \mathrm{W} \\
118^{\circ} 06^{\prime} \mathrm{W} \\
94^{\circ} 40^{\prime} \mathrm{W} \\
? \\
112^{\circ} 25^{\prime} \mathrm{E} \\
112^{\circ} 28^{\prime} \mathrm{E} \\
112^{\circ} 30^{\prime} \mathrm{E} \\
112^{\circ} 20^{\prime} \mathrm{E} \\
112^{\circ} 25^{\prime} \mathrm{E} \\
113^{\circ} 21^{\prime} \mathrm{E} \\
113^{\circ} 08^{\prime} \mathrm{E} \\
100^{\circ} 51^{\prime} \mathrm{E} \\
101^{\circ} 00^{\prime} \mathrm{E} \\
100^{\circ} 00^{\prime} \mathrm{E}\end{array}$ & $\begin{array}{l}70 \\
70 \\
200 \\
20 \\
25 \\
200 \\
100 \\
---- \\
---- \\
750 \\
700 \\
1270 \\
1000 \\
---- \\
---- \\
724 \\
2000 \\
1500\end{array}$ & $\begin{array}{l}2 \\
2 \\
4 \\
-1 \\
-6 \\
5 \\
-12 \\
---- \\
-5 \\
-7 \\
-5 \\
-5 \\
-5 \\
-7 \\
-7 \\
0 \\
0 \\
-5\end{array}$ & $\begin{array}{l}7 \\
7 \\
10 \\
12 \\
10 \\
13 \\
9 \\
---- \\
6 \\
5 \\
6 \\
7 \\
6 \\
5 \\
5 \\
10 \\
8 \\
12\end{array}$ & $\begin{array}{l}660 \\
660 \\
750 \\
1100 \\
1200 \\
550 \\
1200 \\
---- \\
2000 \\
1500 \\
2000 \\
2000 \\
2000 \\
2000 \\
2000 \\
2000 \\
2000 \\
1200\end{array}$ \\
\hline
\end{tabular}

The Pearson correlation coefficient of vein number with cotyledon length, width (W1 and W2) and ratio (R1 and R2) are $0.20,0.07,-0.05,0.13$, and 0.10 , respectively. Cotyledon sizes, especially length, displays a great variation within the species, although it is usually smaller in $C$. yunnanensis than in the other two species (Fig. 5). However, cotyledon shape $(\mathrm{R} 2=\mathrm{W} 1 / \mathrm{W} 2)$ is an ideal character for distinguishing $C$. yunnanensis (linear, mean $\mathrm{R} 2=1.40$ ) from $C$. acuminata var. acuminata (lanceolate, mean $\mathrm{R} 2=2.08$ ) and $C$. lowreyana (lanceolate, mean R2 = 1.98) (see Fig. 5).

\section{Leaves}

Leaf shape (R1, R2, R3) and vein number are relatively consistent within populations and even within species relative to leaf size (length and maximum width). Thus, the leaf is a key character in distinguishing species (Table 6). $C$. lowreyana typically has cordate leaves with a mean of 16 veins on each side, $C$. acuminata usually has oval leaves with fewer than 15 veins on each side, and C. yunnanensis has elliptic leaves with 14 veins Fig. (6). C. lowreyana has a 
Table 3. Means and standard deviations of fruit surface, disc, and size in Camptotheca (Means with the same letter are not significantly different at the 0.001 level. ----- No data available).

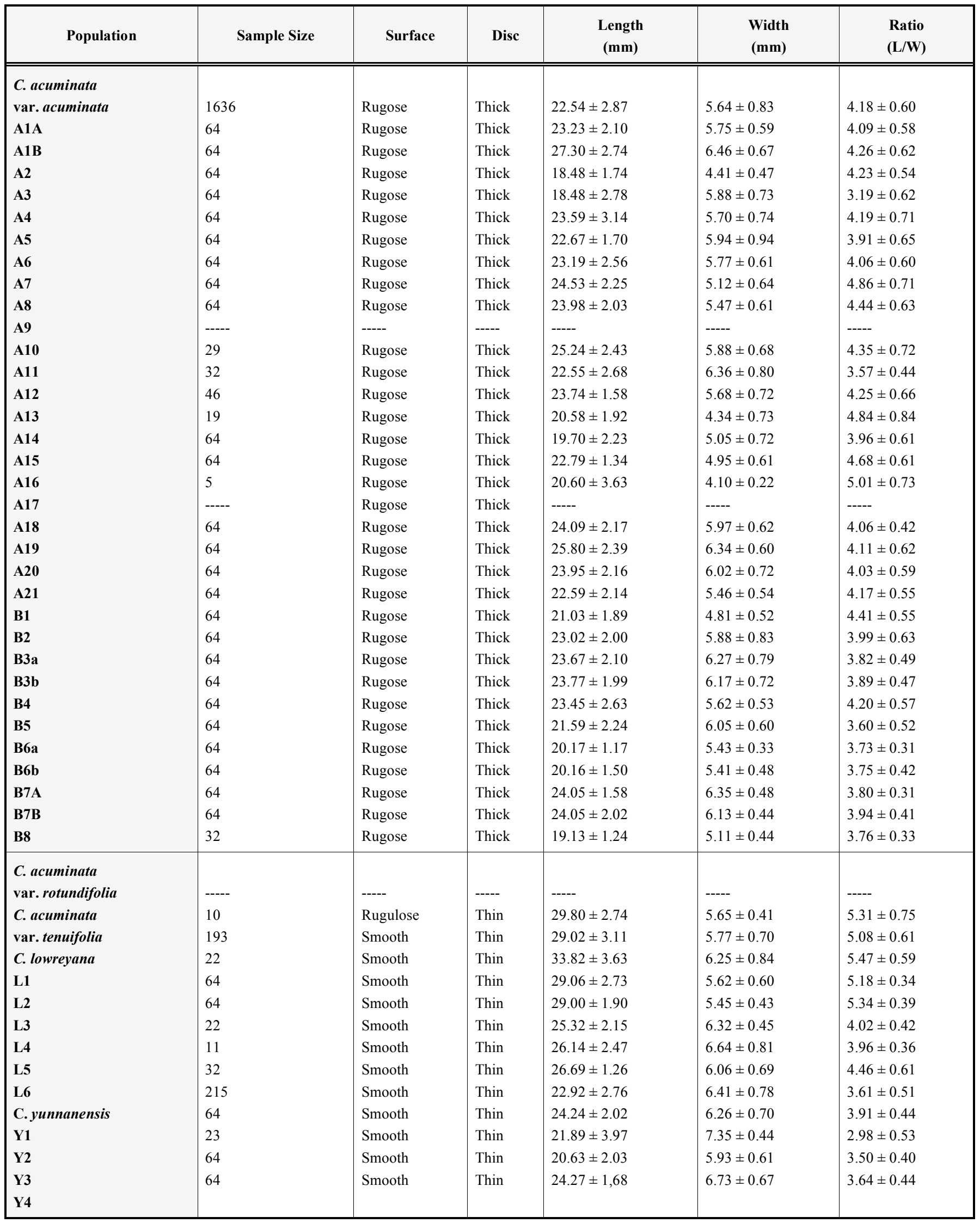




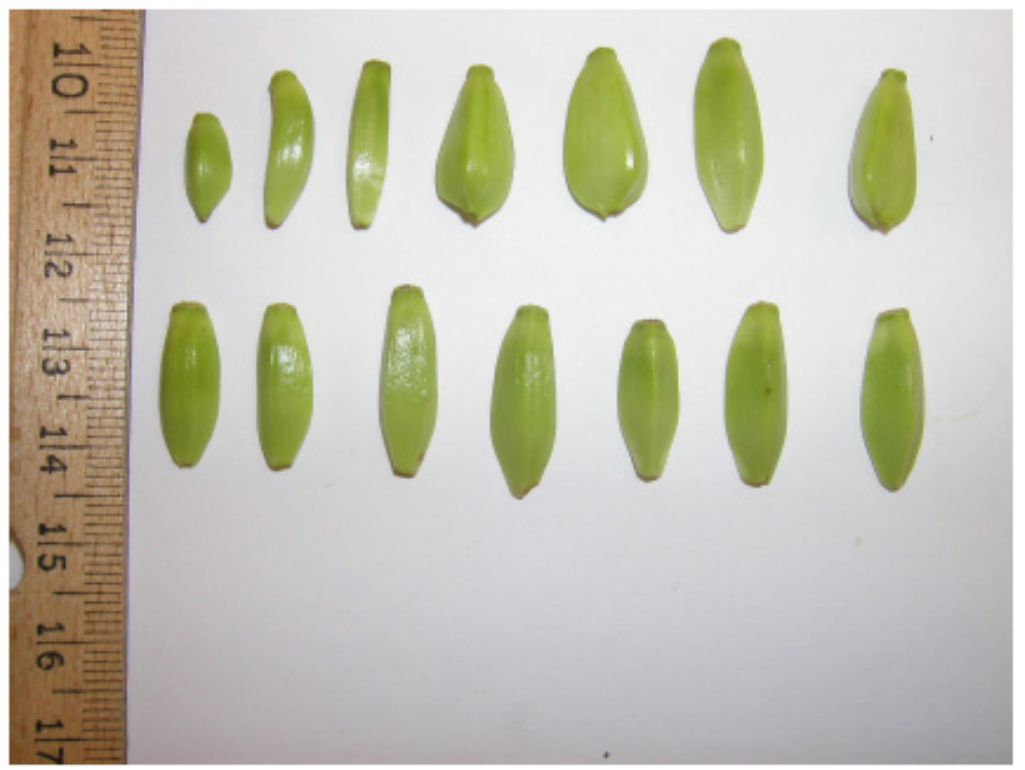

Fig. (2). Variation in mature fruits of Camptotheca acuminata cultivated in Nacogdoches, Texas, USA.

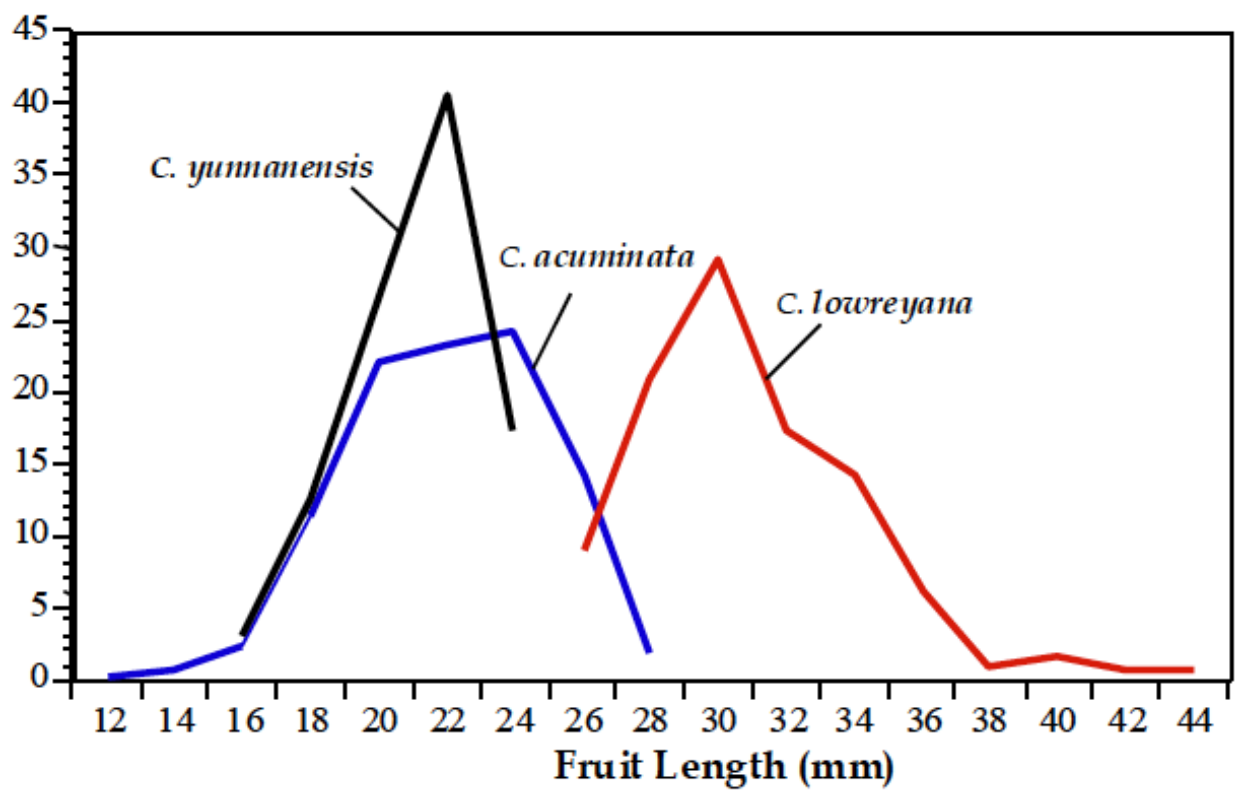

Fig. (3). Frequency distributions of fruit length of C. acuminata $(\mathrm{n}=1283)$, C. lowreyana_ $(\mathrm{n}=193)$, and C. yunnanensis $(\mathrm{n}=87)$.

wider leaf blade base than apex $(\mathrm{R} 2=1.55)$ with the widest point near the blade base $(\mathrm{R} 3=3.37)$, while both $C$. acuminata and $C$. yunnanensis have balanced leaves $(\mathrm{R} 2=1.03$ and 1.18 , respectively) with the widest point in the center of the blade or slightly below center $(\mathrm{R} 3=2.04$ and 2.17 , respectively). However, leaf size, including length and maximum width, is variable with environment and is not a reliable character on which to distinguish the species. Leaf vein number in this study is not strongly related to leaf size; the Pearson correlation coefficient is only 0.54 between vein number and blade length and 0.66 between vein number and blade width.

Leaf margin is not a diagnostic character in distinguishing the taxa of Camptotheca because all three species tend to have serrate leaves in the juvenile phase of development (one or two years old), and gradually become entire after two years (Table 7). This phase variation in leaf margin is not an environmental plasticity due to environmental stimuli; in fact, it is developmental plasticity or fixed phenotypic variation as Bradshaw (1965) noted [7].

\section{DISCUSSION}

\section{Character Variations and Key Characters}

Evolutionary ecologists have stressed the importance of understanding the expression and the components of phenotypic variation among and within populations [1-6] and have proposed that a hierarchical perspective may provide insights into the evolutionary history of a species [3]. 
Table 4. Means and standard deviations of fruit color in Camptotheca (Means with the same letter are not significantly different at the 0.001 level; ----- No data available; general description: R-red, B-brown, and G-gray).

\begin{tabular}{|c|c|c|c|c|c|}
\hline Population & Sample Size & $\begin{array}{l}\text { R.H.S. } \\
\text { Color }\end{array}$ & $\begin{array}{l}\text { Red Color } \\
(1-255)\end{array}$ & $\begin{array}{c}\text { Green Color } \\
(1-255)\end{array}$ & $\begin{array}{c}\text { Blue color } \\
(1-255)\end{array}$ \\
\hline $\begin{array}{l}\text { C. acuminata } \\
\text { var. acuminata } \\
\text { A1A } \\
\text { A1B } \\
\text { A2 } \\
\text { A3 } \\
\text { A4 } \\
\text { A5 } \\
\text { A6 } \\
\text { A7 } \\
\text { A8 } \\
\text { A9 } \\
\text { A10 } \\
\text { A11 } \\
\text { A12 } \\
\text { A13 } \\
\text { A14 } \\
\text { A15 } \\
\text { A16 } \\
\text { A17 } \\
\text { A18 } \\
\text { A19 } \\
\text { A20 } \\
\text { A21 } \\
\text { B1 } \\
\text { B2 } \\
\text { B3a } \\
\text { B3b } \\
\text { B4 } \\
\text { B5 } \\
\text { B6a } \\
\text { B6b } \\
\text { B7 } \\
\text { B8 }\end{array}$ & $\begin{array}{l}450 \\
30 \\
--- \\
30 \\
30 \\
30 \\
30 \\
30 \\
30 \\
30 \\
---- \\
---- \\
--- \\
---- \\
--- \\
---- \\
---- \\
--- \\
--- \\
---- \\
---- \\
---- \\
30 \\
30 \\
30 \\
30 \\
30 \\
30 \\
---- \\
--- \\
---\end{array}$ & $\begin{array}{l}165 \mathrm{~A}-\mathrm{C} \\
165 \mathrm{~B} \\
165 \mathrm{~B} \\
165 \mathrm{~B} \\
165 \mathrm{~B} \\
165 \mathrm{~B} \\
165 \mathrm{~B} \\
165 \mathrm{~B} \\
165 \mathrm{~A} \\
165 \mathrm{~A}-\mathrm{B} \\
---- \\
165 \mathrm{~B} \\
165 \mathrm{C} \\
165 \mathrm{C} \\
165 \mathrm{~B} \\
165 \mathrm{C} \\
165 \mathrm{~B} \\
165 \mathrm{~B} \\
165 \mathrm{~B} \\
165 \mathrm{~B} \\
165 \mathrm{~B} \\
165 \mathrm{~B} \\
165 \mathrm{~B} \\
165 \mathrm{~B} \\
165 \mathrm{~B} \\
165 \mathrm{~B} \\
165 \mathrm{~B} \\
165 \mathrm{~B} \\
165 \mathrm{~B} \\
---- \\
165 \mathrm{~B} \\
165 \mathrm{~A}-\mathrm{B} \\
165 \mathrm{~A}-\mathrm{B}\end{array}$ & $\begin{array}{l}139.03 \pm 23.28 \\
107.97 \pm 9.82 \mathrm{a} \\
---- \\
105.42 \pm 8.80 \mathrm{a} \\
146.27 \pm 10.10 \mathrm{e} \\
118.39 \pm 11.48 \mathrm{~g} \\
150.86 \pm 11.79 \mathrm{de} \\
156.04 \pm 13.27 \mathrm{~cd} \\
115.81 \pm 9.12 \mathrm{~g} \\
117.84 \pm 9.10 \mathrm{~g} \\
---- \\
---- \\
--- \\
--- \\
---- \\
---- \\
---- \\
---- \\
---- \\
---- \\
---- \\
---- \\
---- \\
149.20 \pm 10.12 \mathrm{e} \\
156.85 \pm 10.91 \mathrm{c} \\
163.34 \pm 10.84 \mathrm{~b} \\
165.34 \pm 8.32 \mathrm{~b} \\
165.48 \pm 10.71 \mathrm{~b} \\
133.92 \pm 15.01 \mathrm{f} \\
---- \\
132.79 \pm 8.33 \mathrm{f} \\
---- \\
-\end{array}$ & $\begin{array}{l}102.26 \pm 16.88 \\
84.10 \pm 8.18 \mathrm{a} \\
--- \\
81.74 \pm 7.28 \mathrm{~b} \\
103.28 \pm 7.96 \mathrm{c} \\
88.61 \pm 6.18 \mathrm{~d} \\
110.21 \pm 8.91 \mathrm{e} \\
113.94 \pm 9.78 \mathrm{f} \\
89.01 \pm 6.92 \mathrm{~g} \\
86.83 \pm 6.03 \mathrm{~h} \\
---- \\
---- \\
--- \\
--- \\
--- \\
---- \\
---- \\
---- \\
---- \\
---- \\
---- \\
---- \\
--- \\
104.40 \pm 6.45 \mathrm{i} \\
108.57 \pm 6.78 \mathrm{j} \\
119.74 \pm 7.12 \mathrm{k} \\
135.18 \pm 6.941 \\
119.31 \pm 8.79 \mathrm{~m} \\
97.79 \pm 9.44 \mathrm{n} \\
---- \\
91.15 \pm 6.23 \mathrm{o} \\
--- \\
---\end{array}$ & $\begin{array}{l}60.27 \pm 8.25 \\
53.28 \pm 6.02 \mathrm{a} \\
---- \\
51.38 \pm 5.94 \mathrm{~b} \\
57.89 \pm 5.04 \mathrm{c} \\
55.55 \pm 4.85 \mathrm{~d} \\
68.01 \pm 6.55 \mathrm{e} \\
64.13 \pm 7.10 \mathrm{f} \\
60.67 \pm 6.59 \mathrm{~g} \\
58.67 \pm 7.02 \mathrm{~h} \\
---- \\
---- \\
---- \\
---- \\
---- \\
---- \\
---- \\
---- \\
---- \\
---- \\
---- \\
---- \\
---- \\
61.52 \pm 7.04 \mathrm{i} \\
66.36 \pm 7.11 \mathrm{j} \\
68.69 \pm 7.98 \mathrm{k} \\
61.76 \pm 6.171 \\
59.56 \pm 7.57 \mathrm{~m} \\
62.82 \pm 7.94 \mathrm{n} \\
---- \\
54.02 \pm 6.11 \mathrm{o} \\
---- \\
---\end{array}$ \\
\hline $\begin{array}{l}\text { C. acuminata } \\
\text { var. rotundifolia } \\
\text { C. acuminata } \\
\text { var. tenuifolia } \\
\text { C. lowreyana } \\
\text { L1 } \\
\text { L2 } \\
\text { L3 } \\
\text { L4 } \\
\text { L5 } \\
\text { L6 } \\
\text { C. yunnanensis } \\
\text { Y1 } \\
\text { Y2 } \\
\text { Y3 } \\
\text { Y4 }\end{array}$ & $\begin{array}{l}---- \\
---- \\
30 \\
----- \\
--- \\
30 \\
---- \\
---- \\
----- \\
30\end{array}$ & $\begin{array}{l}---- \\
\text { R-B } \\
\text { 164B-C } \\
164 B \\
164 B-C \\
164 B-C \\
164 B-C \\
164 B-C \\
164 B-C \\
164 B-C \\
164 B \\
164 B-C \\
164 B\end{array}$ & $\begin{array}{l}---- \\
---- \\
137.15 \pm 11.14 \\
---- \\
---- \\
137.15 \pm 11.14 \mathrm{f} \\
---- \\
---- \\
---- \\
179.61 \pm 12.25 \mathrm{~h} \\
----\end{array}$ & $\begin{array}{l}---- \\
---- \\
112.33 \pm 8.78 \\
---- \\
---- \\
112.33 \pm 8.78 p \\
---- \\
---- \\
---- \\
143.93 \pm 10.12 q \\
----\end{array}$ & $\begin{array}{l}----- \\
---- \\
77.82 \pm 7.94 \\
---- \\
---- \\
77.82 \pm 7.94 \text { p } \\
---- \\
---- \\
---- \\
96.07 \pm 8.49 \text { q } \\
-----\end{array}$ \\
\hline
\end{tabular}



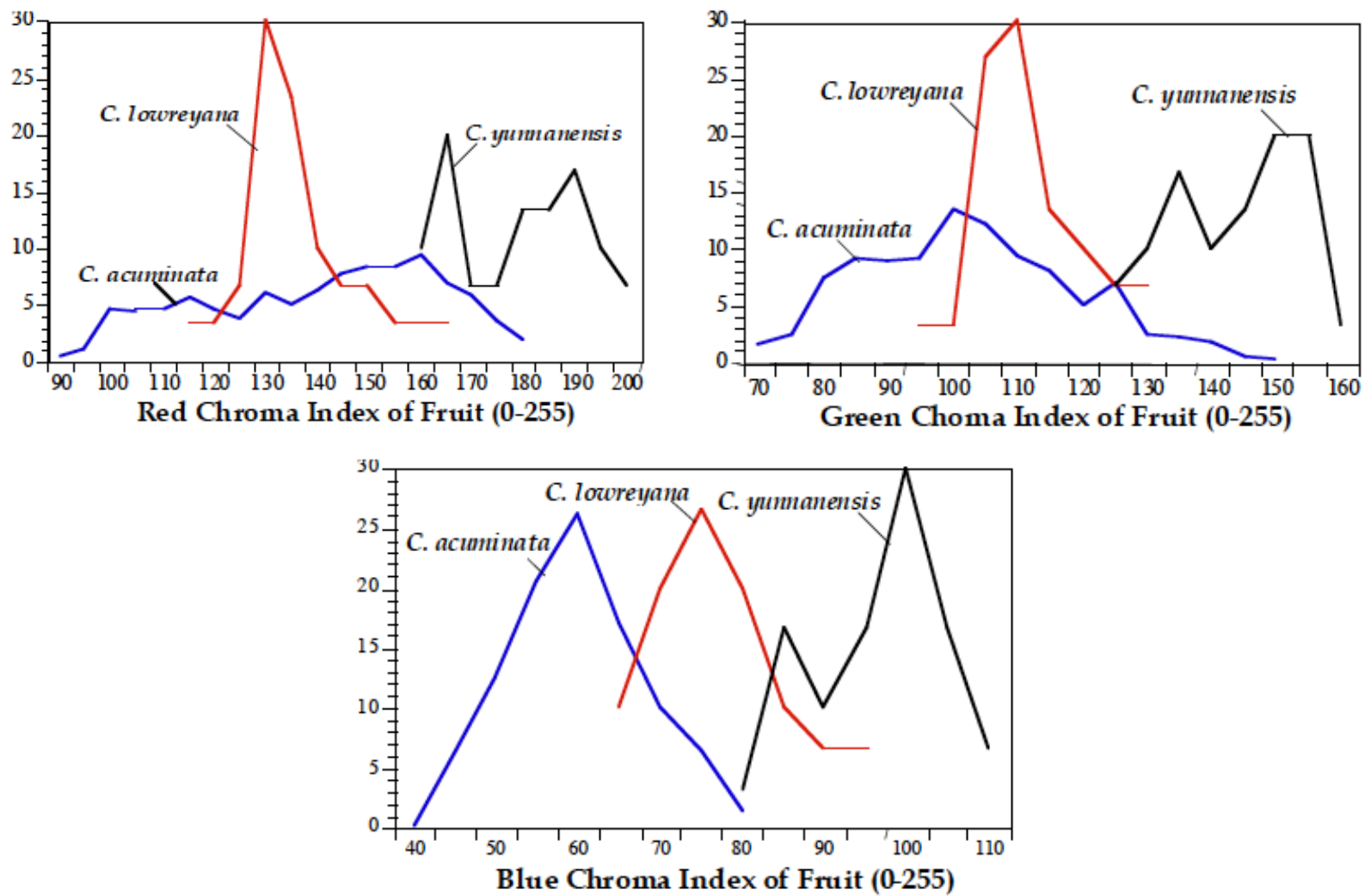

Fig (4). Frequency $(\%$, vertical axis) distributions of fruit color of $C$. acuminata $(\mathrm{n}=450)$, C. lowreyana $(\mathrm{n}=30)$, and $C$. yunnanensis $(\mathrm{n}=$ $30)$.
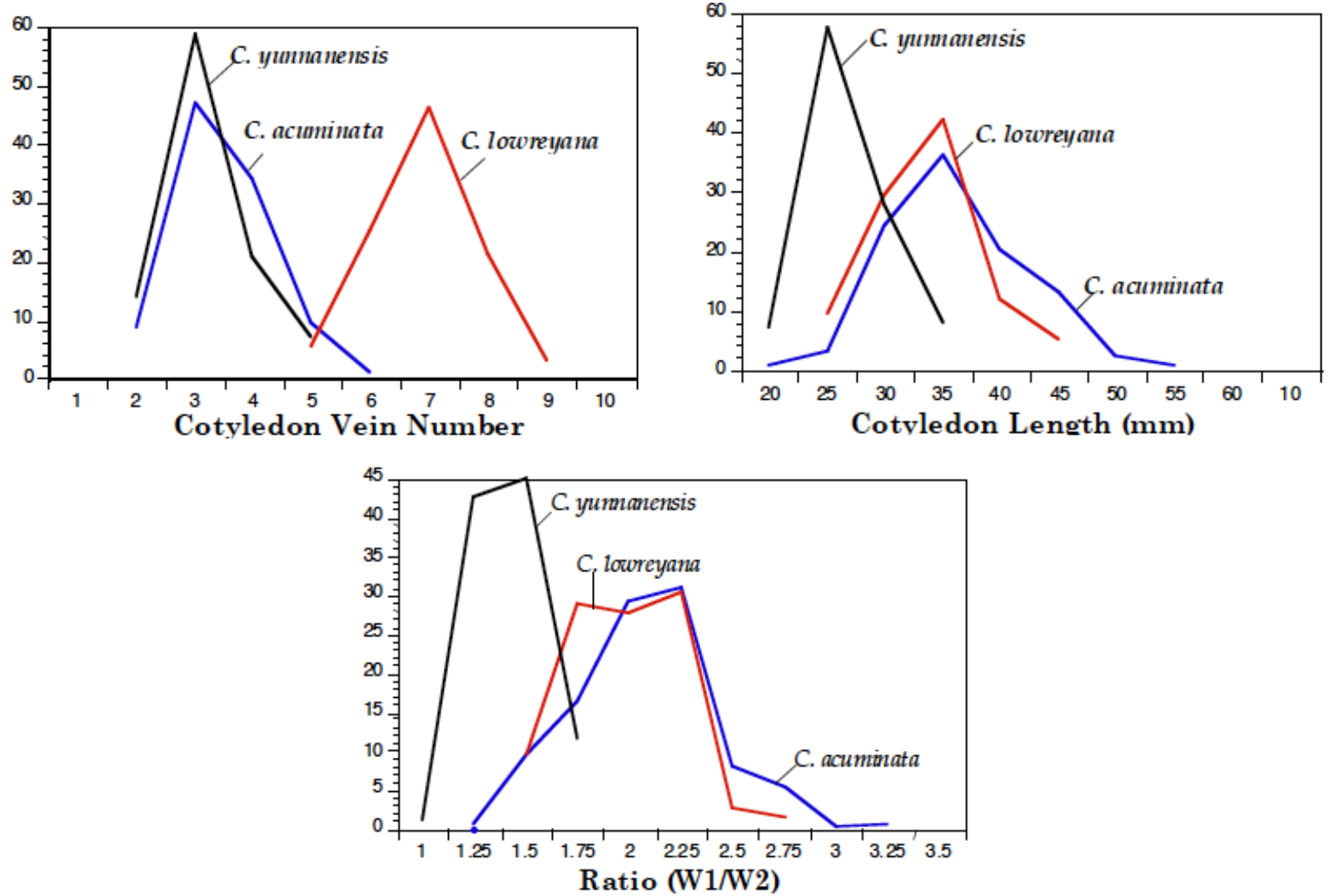

Fig (5). Frequency distributions of cotyledon vein number, length, and size ratio (W1/W2) of C. acuminata $(\mathrm{n}=583)$, C. lowreyana $(\mathrm{n}=76)$, and C. yunnanensis $(\mathrm{n}=87)$. 
Table 5. Means and standard deviations of cotyledon characters in Camptotheca (Means with the same letter are not significantly different at the 0.001 level. * N-pinninerved; P-pinnipalmate. W1 - blade width at 1/5 distance from the base; W2 - blade width at $1 / 5$ distance from the apex).

\begin{tabular}{|c|c|c|c|c|c|c|c|c|}
\hline Population & $\begin{array}{c}\text { Cotyledons } \\
\text { Surveyed }\end{array}$ & $\begin{array}{c}\text { Venation } \\
\text { Type * }\end{array}$ & $\begin{array}{l}\text { Lateral Vein } \\
\text { Number } \\
\text { (each side) }\end{array}$ & $\begin{array}{l}\text { Blade } \\
\text { Length } \\
(\mathrm{cm})\end{array}$ & $\begin{array}{c}\text { W1 } \\
(\mathrm{mm})\end{array}$ & $\begin{array}{c}\text { W2 } \\
(\mathrm{mm})\end{array}$ & $\begin{array}{c}\text { R1 } \\
\text { (Length/W1) }\end{array}$ & $\begin{array}{c}\text { R2 } \\
(\mathrm{W} 1 / \mathrm{W} 2)\end{array}$ \\
\hline A1a & 62 & $P$ & $3.13 \pm 0.74$ & $32.62 \pm 2.86$ & $7.76 \pm 1.03$ & $4.37 \pm 0.82$ & $4.25 \pm 0.43$ & $1.81 \pm 0.29$ \\
\hline A1b & ----- & ----- & 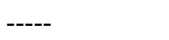 & ---- & ----- & ----- & ---- & ----- \\
\hline A4 & 14 & $\mathrm{P}$ & $3.29 \pm 0.47$ & $30.93 \pm 4.63$ & $6.14 \pm 0.69$ & $2.79 \pm 0.26$ & $5.04 \pm 0.48$ & $2.21 \pm 0.20$ \\
\hline A5 & ----- & ----- & ----- & ----- & ----- & ----- & ----- & ----- \\
\hline A6 & 64 & $\mathrm{P}$ & $4.19 \pm 0.77$ & $44.13 \pm 4.66$ & $7.93 \pm 1.15$ & $3.70 \pm 0.69$ & $5.63 \pm 0.68$ & $2.19 \pm 0.36$ \\
\hline A7 & 80 & $\mathrm{P}$ & $3.85 \pm 0.81$ & $39.88 \pm 4.03$ & $8.73 \pm 1.25$ & $4.24 \pm 0.55$ & $4.63 \pm 0.55$ & $2.07 \pm 0.30$ \\
\hline $\mathbf{A 8}$ & 64 & $\mathrm{P}$ & $3.59 \pm 0.68$ & $40.13 \pm 4.34$ & $7.11 \pm 0.82$ & $3.52 \pm 0.46$ & $5.68 \pm 0.57$ & $2.05 \pm 0.29$ \\
\hline A12 & ----- & ----- & ----- & ----- & ----- & ----- & ----- & ----- \\
\hline A13 & ----- & ----- & ----- & ----- & ----- & ----- & ----- & ----- \\
\hline A14 & ---- & ----- & ----- & ----- & ----- & ---- & ----- & ----- \\
\hline A15 & ----- & ----- & ----- & ----- & ----- & ----- & ----- & ----- \\
\hline A16 & ----- & ----- & ----- & ----- & ----- & ----- & ----- & ----- \\
\hline A17 & ----- & ----- & ----- & ----- & ----- & ----- & ----- & ----- \\
\hline A18 & ----- & ----- & ----- & ----- & ----- & ----- & ----- & ----- \\
\hline A19 & ----- & ----- & ----- & ----- & ----- & ----- & ----- & ----- \\
\hline A20 & ----- & ----- & ----- & ----- & ----- & ----- & ----- & ----- \\
\hline A21 & ---- & ----- & ----- & ----- & ----- & ---- & ----- & ----- \\
\hline B6b & 84 & $\mathrm{P}$ & $3.67 \pm 0.92$ & $33.61 \pm 4.46$ & $7.93 \pm 0.65$ & $3.75 \pm 0.49$ & $4.26 \pm 0.61$ & $2.14 \pm 0.23$ \\
\hline B7a & ----- & ----- & ----- & ----- & ----- & ----- & ----- & ----- \\
\hline B7b & ----- & ----- & ----- & ---- & ---- & ----- & ---- & ----- \\
\hline B8 & ---- & ----- & ----- & ----- & ---- & ----- & ----- & ----- \\
\hline C. acuminata var. rotundifolia & ----- & ----- & ----- & ----- & ----- & ----- & ----- & ----- \\
\hline C. acuminata var. tenuifolia & ---- & ---- & ----- & ----- & ----- & ----- & ----- & ---- \\
\hline C. lowreyana & 76 & $\mathrm{~N}$ & $6.91 \pm 0.88$ & $34.29 \pm 4.93$ & $7.46 \pm 1.32$ & $3.80 \pm 0.70$ & $4.66 \pm 0.61$ & $1.98 \pm 0.27$ \\
\hline L1 & ----- & ----- & ----- & 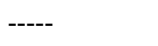 & ----- & ----- & ----- & ----- \\
\hline $\mathbf{L 2}$ & 27 & $\mathrm{~N}$ & $7.07 \pm 0.78$ & $33.78 \pm 4.15$ & $7.04 \pm 1.17$ & $3.52 \pm 0.56$ & $4.87 \pm 0.70$ & $2.01 \pm 0.24$ \\
\hline $\mathbf{L 3}$ & 49 & $\mathrm{~N}$ & $6.82 \pm 0.93$ & $34.57 \pm 5.32$ & $7.69 \pm 1.36$ & $3.96 \pm 0.72$ & $4.54 \pm 0.53$ & $1.96 \pm 0.28$ \\
\hline L4 & ----- & ----- & ----- & ----- & ----- & ----- & ----- & ----- \\
\hline L5 & ----- & ----- & ----- & ----- & ----- & ----- & ----- & ----- \\
\hline L6 & ----- & ---- & ----- & ---- & ---- & ---- & ---- & ---- \\
\hline C. yunnanensis & 87 & $\mathrm{P}$ & $3.21 \pm 0.76$ & $26.92 \pm 3.29$ & $5.37 \pm 0.81$ & $3.85 \pm 0.58$ & $5.07 \pm 0.67$ & $1.40 \pm 0.16$ \\
\hline Y1 & ----- & ----- & ----- & ----- & ----- & ----- & ----- & ----- \\
\hline Y2 & ----- & ----- & ----- & ----- & ----- & ----- & ----- & ----- \\
\hline Y3 & 87 & $\mathrm{P}$ & $3.21 \pm 0.76$ & $26.92 \pm 3.29$ & $5.37 \pm 0.81$ & $3.85 \pm 0.58$ & $5.07 \pm 0.67$ & $1.40 \pm 0.16$ \\
\hline Y4 & ----- & ----- & ----- & ----- & ----- & ----- & ----- & ----- \\
\hline
\end{tabular}


Table 6. Means and standard deviations of leaf characters in Camptotheca (Means with the same letter are not significantly different at the 0.001 level. * $\mathrm{C}$-cordate, $\mathrm{E}$-elliptic, $\mathrm{NE}$-narrowly elliptic, Ol—oval, $\mathrm{R}$-round, Ot-ovate; **according to picture/publication. $* *$ data from three parent trees. ---- no data available. $\mathrm{W} 1$ - blade width at $1 / 5$ distance from the base; W2 - blade width at 1/5 distance from the apex; Lm-distance of the widest point from the base along the main vein).

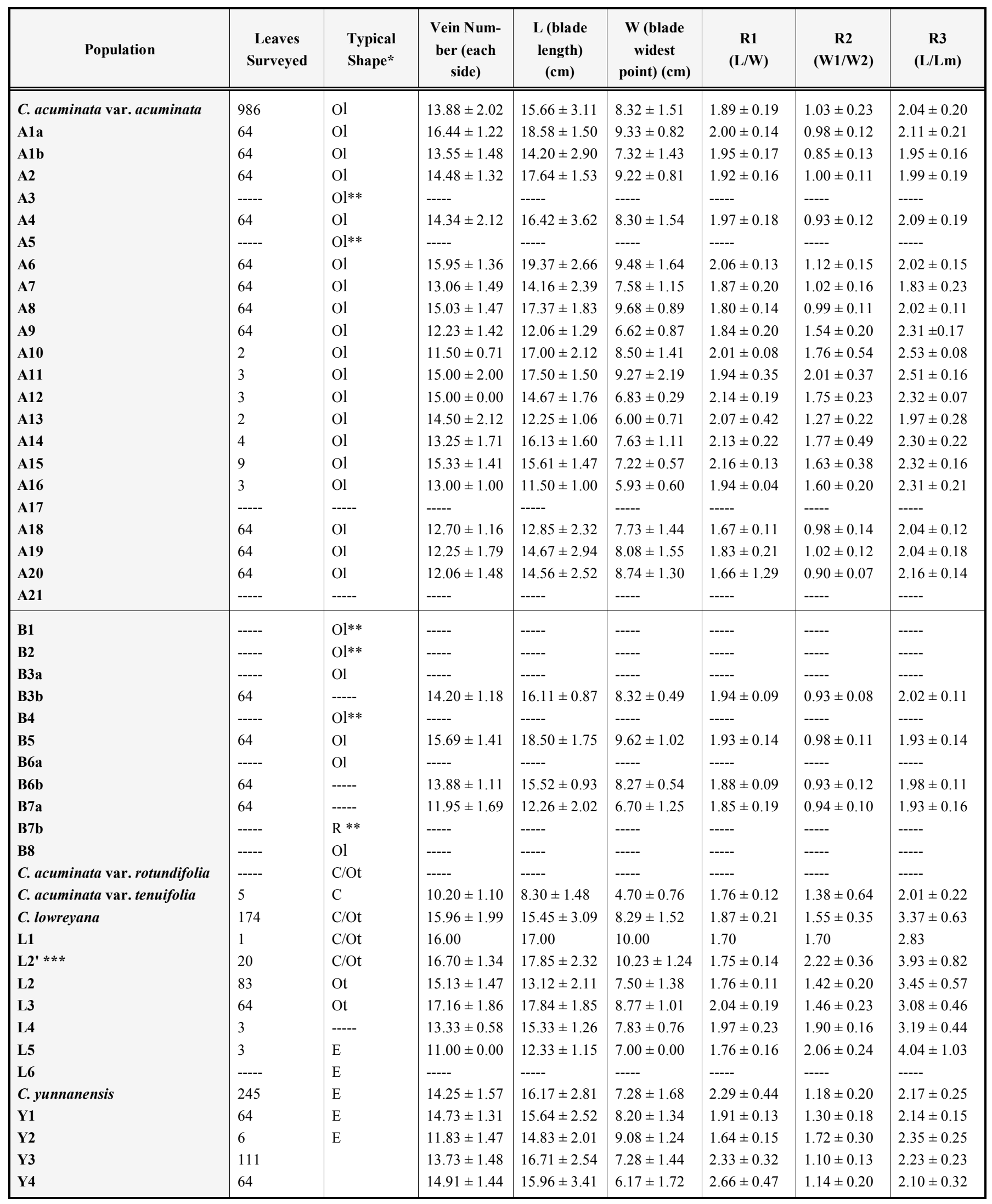




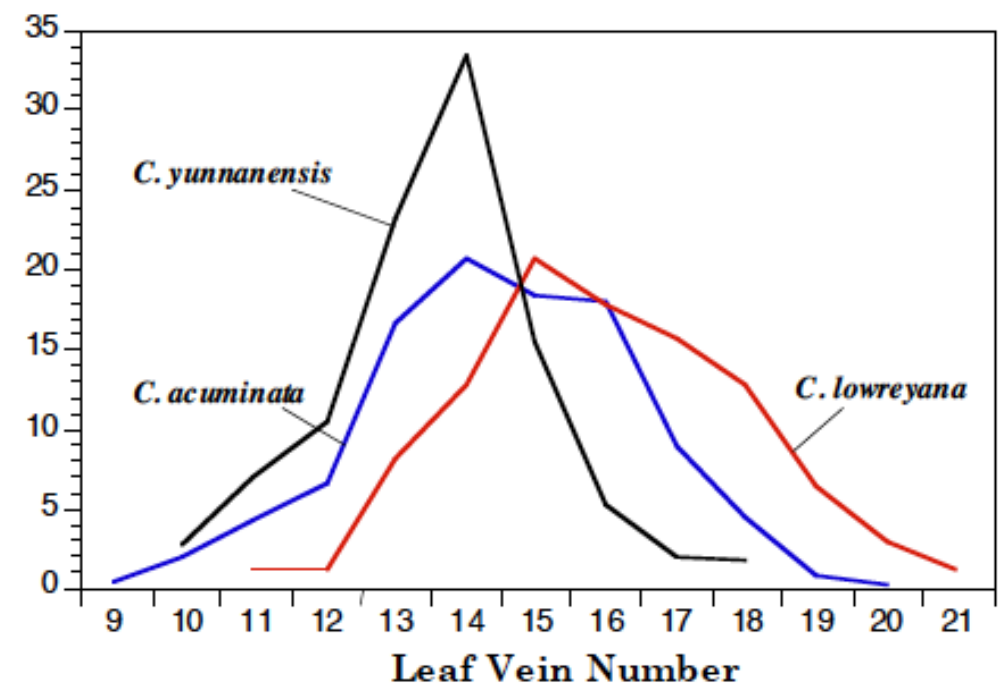

Fig (6). Frequency distributions of leaf vein number of $C$. acuminata_ $(\mathrm{n}=986)$, C. lowreyana $(\mathrm{n}=174)$, and C. yunnanensis $(\mathrm{n}=245)$.

Seed size is a critical feature in the life history of a plant [8]. The size of a seed provides a measure of the size of the nutrient reserve provided for the embryo by the parent. Thus, seed size affects not only the dispersal, but also the establishment of seedlings. Seed size within a species is generally regarded as relatively constant $[9,10]$. However, considerable variation in seed size is still widely observed among and within populations, within single plants, and even among years in the same plant in many species $[2,11]$.

The fruit of Camptotheca is flattened and samara-like, containing only a single seed. Thus, the fruit of Camptotheca is treated the same as any other seed (or fruit). The fruit surface texture and disc are consistent within the populations and species studied.

The cotyledon is a conservative organ in plant life history and is important and often overlooked in plant taxonomy and phylogeny $[12,13]$. Cotyledon shape and venation pattern is usually relatively stable within populations and within species and can provide important diagnostic characters for distinguishing the species in some genera $[12,14]$.

The leaf is commonly used for plant identification. Seedling leaves are also important to Camptotheca because of their high concentration of CPTs [15].

Our phenotypic analysis showed that key diagnostic characters for the identification of taxa within Camptotheca include leaf shape, venation type, vein number, stoma size and frequency, outer stomatal rim, subsidiary cell number, and gland size (Table 8); cotyledon shape, venation type, and vein number; and the fruit surface texture, disc, length, and color. Other characters could not be shown to be dependable for diagnostic purposes.

\section{Variation within and Among Populations}

Camptotheca is a polygamo-monoecious genus. Neither self-fertilization nor agamospermy were observed in C. acuminata var. acuminata [16] because the stamens are shed nearly one week before the stigma of the same flower becomes receptive. Therefore, cross-pollination is the major breeding system of the species. Indeed, pollination of Camp- totheca is obligately entomophilous, and no fruit is produced in the absence of visiting insects (bees, butterflies, flies, and beetles) [16]. Seed set depends on the activities of pollinating insects. Species cross-pollinated by insects generally have a greater proportion of their variation within rather than among populations [17]. Major phenotypic variation was observed among populations for most measures (Table 9). The current population structure and distribution of existing trees may largely shape this variation pattern. Camptotheca has a fragmented geographic distribution and populations are well isolated from each other. Each natural population is very small, from 1-10 mature trees according to our observations. Selfing (crossing between male and female structures of the same plant) or related matings (crossing between closely related individual plants) are greatly increased due to the small sizes of populations.

Relatedness is also common in plantations as the plants frequently show the same seed source and may even be ramets of the same clone. Consequently, the variation in both wild populations and plantation trees now existing in China is narrow. The extent of the variation base among trees cultivated in the United States is very narrow. Most of the mature trees can be traced back to the same provenance, and all are solitary except in Chico, California, where two trees are known to exist. Therefore, selfing is the only breeding system for nearly all individual trees in the United States. Consequently, all existing plants received their limited gene pool from parents displaying obvious heterozygosity loss for generations. If the effective population size of Camptotheca is 20 under natural conditions, genetic variation will decrease by $2.5 \%$ per generation according to Koenig's model $1 / 2 \mathrm{~N}$ (1988).

A majority of the observed phenotypic variation for most measures was among populations. In addition, related matings or selfing (the most severe form of related matings) is common in both natural and cultivated populations of Camptotheca. Therefore, it is not possible to broaden genetic diversity at the species level using only materials from a single population. To conserve genetic diversity of Camptotheca, it is necessary to collect seeds from all populations. 
Table 7. Leaf margin characters in Camptotheca (* according to the original description. ----- No data available).

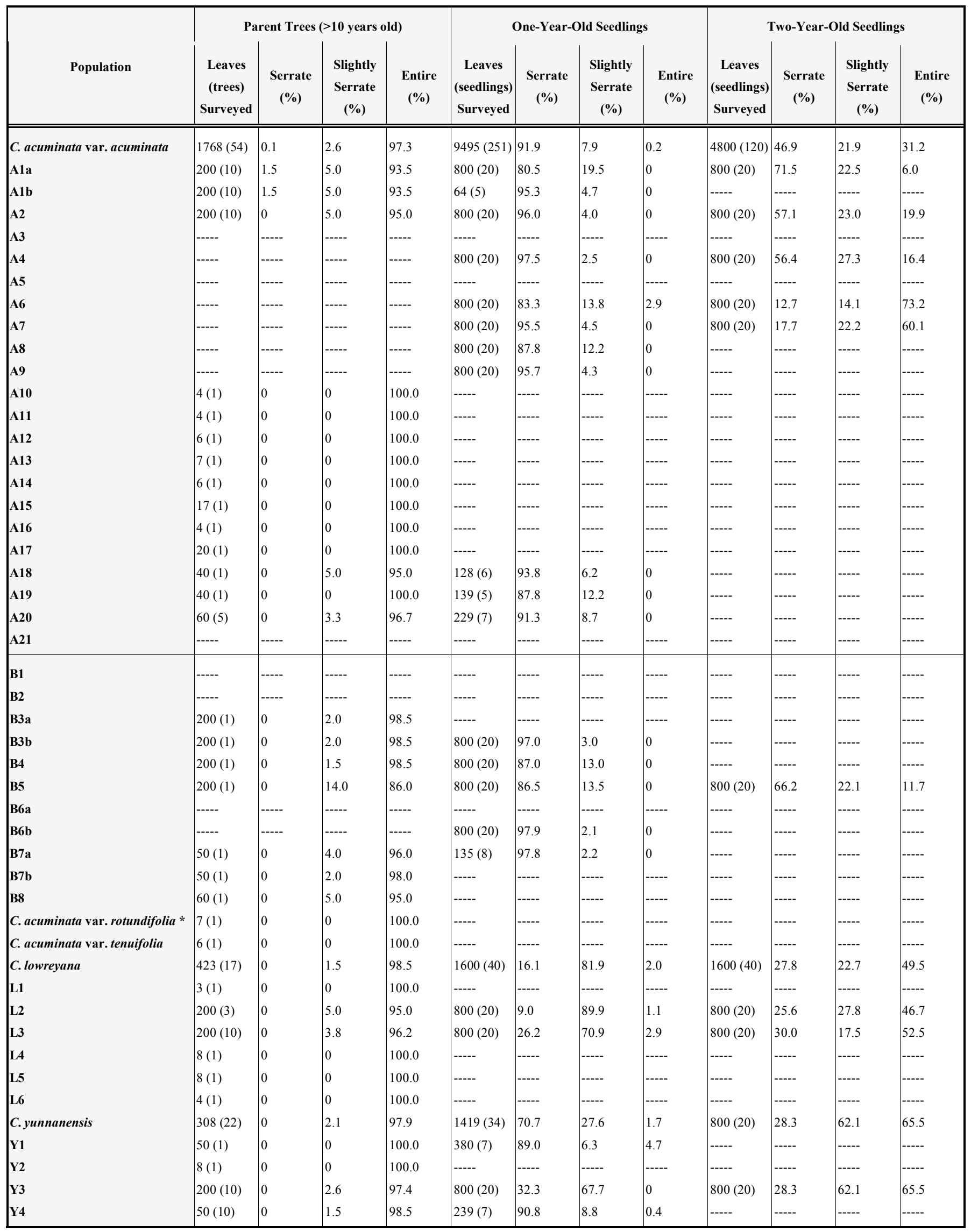


Table 8. Means and standard deviations of leaf stoma features in Camptotheca (Means with the same letter are not significantly different at the 0.001 level. * 120 cells were measured for each population).

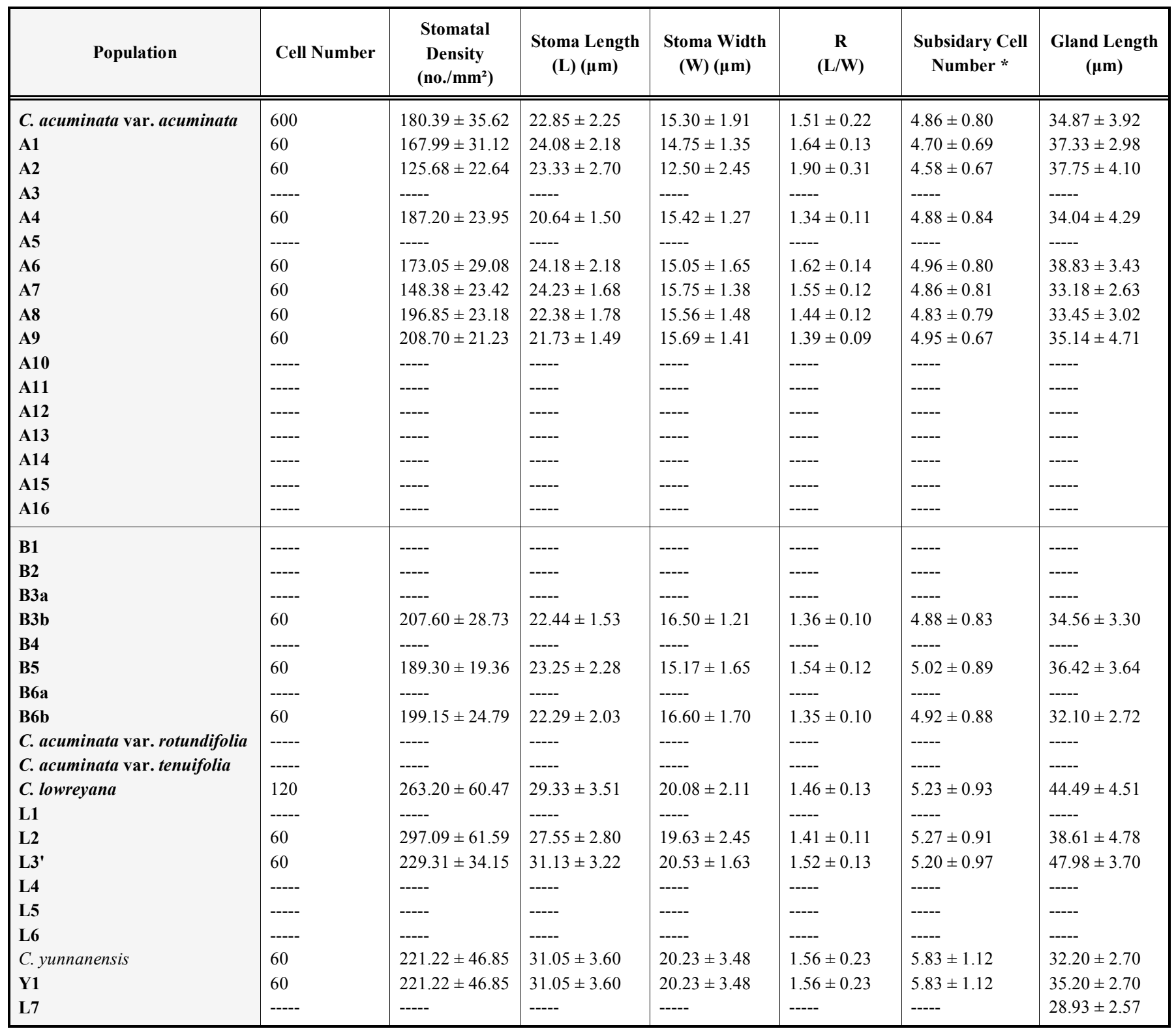

\section{Variations Among Generations}

Reproduction from seed is a sexual process that results in genetically variable offspring (Raulston and Tripp 1994). Any population of seedlings demonstrates an amazing array of variability. However, some populations exhibit more phenotypic variation than others do.

Six samples of $C$. acuminata var. acuminata in the United States represent four selfing generations from the same provenance (first generation: B1, second: B3, third: B4 and B11, and fourth: B5 and B10). B6 and B7 are two selfing generations from the tree in Honolulu, Hawaii. Each of these eight samples and B1 share the same parental base from the Guangxi or Sichuan provenance (Steward No. 75).

Under different environmental conditions (see Table 1), the composite samples from these trees are close in pedigree and are more similar to each other than other samples in both means and frequency distributions of leaf vein number, fruit length, fruit colors, and cotyledon vein number (see Tables 3-6). For example, B2, B3, and B4 are similar to each other, and relatively similar to $\mathrm{B} 1$ and $\mathrm{B} 6$, but less similar to others (e.g., A2 and A6) in fruit length (23.03-23.67 vs. 21.03 of B1 and 20.17 of B6 vs. 23.19 of A6 and 18.48 of A2 and A3), red chroma (156.85-165.48 vs. 149.20 of B1 and 133.92 of B6 vs. 115.81 of A6 and 105.42 of A2) and green chroma (108.57-119.74 vs. 104.40 of B1 and 97.79 of B6 vs. 89.01 of A6 and 81.74 of A2). These data indicate that fruit length and chroma is controlled genetically from generation to generation and is only marginally affected, if at all, by local physical environment.

A major factor contributing to variation in fruit length and chroma is the efficiency of pollination. The reason that no significant variation in fruit length and chroma was 
Table 9. Tukey's Studentized Range (HSD) pairwise absolute mean differences for each measure between taxa (W1-blade width of cotyledon at $5 \mathrm{~mm}$ from the base; W2-blade width of cotyledon at $5 \mathrm{~mm}$ from the apex. Level of significance: $* *$ at $P<0.01 ; * * *$ at $P<0.001$; ns: not significant at $P<0.05$. ---- No data available. The difference between means listed in the table is measured as column - row).

\begin{tabular}{|c|c|c|c|}
\hline C. lowreyana & $\begin{array}{l}\text { Leaf vein number } \\
\text { Leaf blade length (LL) } \\
\text { Leaf blade width (LW) } \\
\text { Leaf ratio (LW/LL) } \\
\text { Fruit length (FL) } \\
\text { Fruit width (FW) } \\
\text { Fruit ratio (FW/FL) } \\
\text { Fruit red color } \\
\text { Fruit green color } \\
\text { Fruit blue color } \\
\text { Cotyledon vein number } \\
\text { Cotyledon length (CL) } \\
\text { Cotyledon width (W1) } \\
\text { Cotyledon width (W2) } \\
\text { Cotyledon ratio R1 (CL/W1) } \\
\text { Cotyledon ratio R2 (W1/W2) }\end{array}$ & $\begin{array}{l}3.51 * * * \\
1.99 * * * \\
1.25 * * * \\
0.01 * * * \\
8.23 * * * \\
0.14 * * * \\
0.07 * * * \\
\mathrm{~ns} \\
10.07 * * * \\
17.55 * * * \\
3.43 * * * \\
-1.93^{* * *} \\
-0.49 * * * \\
\mathrm{~ns} \\
\mathrm{~ns} \\
\mathrm{~ns}\end{array}$ & \\
\hline C. yunnanensis & $\begin{array}{l}\text { Leaf vein number } \\
\text { Leaf blade length (LL) } \\
\text { Leaf blade width (LW) } \\
\text { Leaf ratio (LW/LL) } \\
\text { Fruit length (FL) } \\
\text { Fruit width (FW) } \\
\text { Fruit ratio (FW/FL) } \\
\text { Fruit red color } \\
\text { Fruit green color } \\
\text { Fruit blue color } \\
\text { Cotyledon vein number } \\
\text { Cotyledon length (CL) } \\
\text { Cotyledon width (W1) } \\
\text { Cotyledon width (W2) } \\
\text { Cotyledon ratio R1 (W1/CL) } \\
\text { Cotyledon ratio R2 (W2/W1) }\end{array}$ & $\begin{array}{l}\mathrm{ns} \\
\mathrm{ns} \\
1.98 * * * \\
0.12 * * * \\
1.50 * * * \\
0.33 * * * \\
0.03 * * * \\
40.57 * * * \\
41.67 * * * \\
35.80 * * * \\
-0.27 * * * \\
-9.35 * * * \\
-2.58 * * * \\
\mathrm{~ns} \\
0.43 * * * \\
-0.67 * * *\end{array}$ & $\begin{array}{l}3.43 * * * \\
2.17 * * * \\
3.23^{* * *} \\
0.13^{* * *} \\
9.73^{* * *} \\
\mathrm{~ns} \\
0.10^{* * *} \\
42.45^{* * *} \\
31.60^{* * *} \\
18.25^{* * *} \\
-3.70^{* * *} \\
-7.36 * * * \\
-2.09 * * * \\
\mathrm{~ns} \\
0.41 * * * \\
-0.57 * * *\end{array}$ \\
\hline
\end{tabular}

observed among Chico (B2), San Antonio (B3), and Kingwood (B4) trees is that the pollination pool of $C$. acuminata var. acuminata was fixed in these three locations. In both San Antonio and Kingwood, there was only a single mature C. acuminata var. acuminata tree in 1993 and 1994 when fruits were collected. Chico had only two mature trees during this period. Thus, it is expected that selfing or related matings restricted the genetic variation within those observed plants.

\section{Variation Among Populations in the Same Habitat and Location}

Both A1 and L3 samples came from the same habitat and the same general location. The distance between the closest trees within these two populations is about $200 \mathrm{~m}$. L3 had a mean fruit size of $29.00 \mathrm{~cm}$ in length, $5.45 \mathrm{~mm}$ in width, and 5.34 in ratio, while A1A had a mean fruit size of $23.23 \mathrm{~cm}$ in length, $5.75 \mathrm{~mm}$ in width, and 4.09 in ratio (see Table 3). They also show significant differences in fruit color and tex- ture: fruits of L3 have a smooth surface and gray-brown color (chroma indices of 137.15 in red, 112.33 in green, and 77.82 in blue) while those of A1 have a rugose surface and are red-brown in color (107.97 in red, 84.10 in green, and 53.28 in blue) (see Table 5). In addition, samples from each of the two populations differ in cotyledon features, especially venation type and vein number (see Table 5).

Camptotheca acuminata var. acuminata is commonly planted in the "four-sides" (waterside, hillside, roadside, and homeside); however, seed sources are widely scattered and dependent on where mature surviving natural trees happen to be found. Provenance tests for optimum ecotype selections have never been done and seed is not commercially available.

It is generally expected that outcrossing plants like Camptotheca have small variations among populations and large within populations. However, our study found that a majority of the observed phenotypic variation was among populations for most morphometric characters (Table 10). 
Table 10. Sources of morphological variations for the genus Camptotheca (W1-blade width of cotyledon at $5 \mathrm{~mm}$ from the base; W2blade width of cotyledon at $5 \mathrm{~mm}$ from the apex; VWP-variation within populations; VAP-variation among population; VAS-variation among species).

\begin{tabular}{|c|c|c|c|}
\hline Character & $\begin{array}{c}\text { VWP } \\
\text { (\% of total) }\end{array}$ & $\begin{array}{c}\text { VAP } \\
\text { (\% of total) }\end{array}$ & $\begin{array}{c}\text { VAS } \\
(\% \text { of VAP) }\end{array}$ \\
\hline Leaf vein number & 5.46 & 94.54 & 30.35 \\
\hline Leaf blade length (LL) & 31.59 & 68.41 & 6.65 \\
\hline Leaf blade width (LW) & 17.07 & 82.93 & 21.91 \\
\hline Fruit length (FL) & 25.91 & 74.09 & 73.30 \\
\hline Fruit width (FW) & 63.23 & 36.77 & 3.31 \\
\hline Fruit ratio (FW/FL) & 45.72 & 54.28 & 55.31 \\
\hline Fruit red color & 23.42 & 76.58 & 25.43 \\
\hline Cotyledon vein number & 25.32 & 74.68 & 99.00 \\
\hline Cotyledon length (CL) & 12.93 & 87.07 & 27.45 \\
\hline Cotyledon width (W1) & 45.80 & 54.20 & 97.81 \\
\hline Cotyledon width (W2) & 86.73 & 13.27 & 90.73 \\
\hline Cotyledon R1 (W1/CL) & 57.94 & 42.06 & 87.27 \\
\hline Cotyledon R2 (W2/W1) & 39.00 & 61.00 & 91.37 \\
\hline
\end{tabular}

This is probably due largely to selfing and related matings common to both natural and cultivated populations, resulting in the current population structure that now displays a highly fragmented distribution pattern. This makes it difficult to extend patterns from a single population to the species level in the genus. For genetic resource conservation, it is necessary to collect data from as many populations with as few individuals within the population as possible in order to ensure greater genetic diversity. This is true even if the population consists of only one tree. This means that conservation of all remaining wild trees is imperative. A recommendation to that effect has been submitted to the Chinese government.

As a result of taxonomic treatment based on key characters, the taxon from Yunnan is treated as C. yunnanensis. The newly described species, $C$. lowreyana, produces large fruit and high CPT yield. This makes C. lowreyana an important candidate for plantation development because drug extract levels normally are greatest in the fruit ( $\mathrm{Li}$ and Adair 1994). C. yunnanensis has a long growing period; this is a characteristic which may make C. yunnanensis an important selection for biomass production. The plantation possibilities of both taxa will be explored in future studies.

\section{CONFLICT OF INTEREST}

The authors confirm that this article content has no conflict of interest.

\section{ACKNOWLEDGEMENTS}

Declared none.

\section{PATIENT'S CONSENT}

Declared none.

\section{REFERENCES}

[1] Primack, R. B.; Kang, H. Measuring fitness and natural selection in wild plant populations. Ann. Rev. Ecol. Syst., 1989, 20, 367-396.

[2] Wolf, L. L.; Hainsworth, F. R.; Mercier, T.; Benjiamin, B. Seed size variation and pollinator uncertainty in Ipomopsis aggregata (Polemoniaceae). J. Ecol., 1986, 74, 361-371.

[3] Delesalle, V. A.; Mazer, S. J. The structure of phenotypic variation in gender and floral traits within and among populations of Spergularia marina. Am. J. Bot., 1995, 82, 798-810.

[4] Montagnes, R. J. S.; Vitt, D. H. Patterns of phenotypic variation in Meesia triquetra (Bryopsida: Meesiaceae) over an Arctic-Boreal gradient. Syst. Bot., 1991, 16, 726-735.

[5] Mitchell-Olds, T. Quantitative genetics of survival and growth in Impatiens capensis. Evolution, 1986, 40, 107-116.

[6] Venable, D. L. Using intraspecific variation to study the ecological significance and evolution of plant life-histories. In: Perspectives on Plant Population Ecology; Dirzo, R.; Sarukhan, J., Eds; Sinauer: Sunderland, Massachutts, 1981; pp. 166-187.

[7] Bradshaw, A. D. Evolutionary significance of phenotypic plasticity in plants. Adv. Genet., 1965, 13, 115-155.

[8] Leshman, M. R.; Westoby, M. Hypotheses on seed size: tests using the semiarid flora of western New South Wales, Australia. Am. Nat., 1994, 143, 890-906. 
[9] Stebbins, G. L. Adaptive radiation of reproductive characteristics in angiosperms. II. seeds and seedlings. Ann. Rev. Ecol. Syst., 1971, 2, 237-260.

[10] Harper, J. L. Population biology of plant. Academic Press: London, 1994.

[11] Sakai, S.; Sakai, A. Flower size-dependent variation in seed size: theory and a test. Am. Nat., 1985, 145, 918-934.

[12] Zhang, R. H.; Liu, H. E.; Wang, Z. T. Seedling morphology of important trees in China. Science Press: Beijing, 1993.

[13] Huang, P.H. Evolution trends and relationships of some traits from the view of seedling morphology. J. N.E. For. Univ., 1980, 36-56.
[14] Li, S. Y.; Ning, Z. Morphology, taxonomy and distribution of the genus Rhamnus L. (Rhamnaceae) in the northeastern China. Bull. Bot. Res., 1988, 8(2), 71-114.

[15] Li, S. Y.; Yi, Y. J.; Wang, Y. J.; Zhang, Z. Z.; Beasley, R. S. Camptothecin accumulation and variation in Camptotheca Decaisne. Planta Med., 2002, 68(11), 1010-1016.

[16] Chen, L. J.; wang, F. H.; Wu, Y. R. The pollination biology of Camptotheca acuminata Decne. (Nyssaceae). Cathaya, 1991, 3, 45-52.

[17] Hamrick, J. L.; Godt, M. J. Allozyme diversity in plant species. In Plant population genetics, breeding, and genetic resources, Brown, A. D.; Clegg, M. T.; Kahler, A. L.; Weir, B. S., Eds. Sinauer Sunderland, Massachusetts, 1990, pp. 43-63.

Received: December 30, 2013

Revised: September 16, 2014

Accepted: September 24, 2014

(C) Shiyou Li; Licensee Bentham Open.

This is an open access article licensed under the terms of the Creative Commons Attribution Non-Commercial License (http://creativecommons.org/licenses/by-nc/3.0/) which permits unrestricted, non-commercial use, distribution and reproduction in any medium, provided the work is properly cited. 